PROCEEDINGS OF THE

AMERICAN MATHEMATICAL SOCIETY

Volume 130, Number 12, Pages 3713-3717

S 0002-9939(02)06487-0

Article electronically published on May 14, 2002

\title{
SOFT ALMOST DISJOINT FAMILIES
}

\author{
PAUL J. SZEPTYCKI
}

(Communicated by Alan Dow)

\begin{abstract}
An almost disjoint family $A$ is said to be soft if there is an infinite set that meets each $a \in A$ in a nonempty but finite set. We consider the associated cardinal invariant defined to be the minimal cardinality of an almost disjoint family that is not soft. We show that this cardinal coincides with J. Brendle's cardinal ap.
\end{abstract}

\section{INTRODUCTION}

We will say that an almost disjoint family $A \subseteq[\omega]^{\aleph_{0}}$ is soft if there is an infinite $X \subseteq \omega$ such that $0<|X \cap a|<\omega$ for each $a \in A$. This definition, introduced by J. E. Vaughan, arose in the study of property (a) in the context of $\Psi$-like spaces (see [3). A topological space $X$ is said to have property $(a)$ if for each open cover $\mathcal{U}$ and each dense subset $D \subseteq X$ there is a closed discrete $C \subseteq D$ such that $\{U \in \mathcal{U}: U \cap C \neq \emptyset\}$ covers $X$. If $A$ is an almost disjoint family, $\Psi(A)$ denotes the Mrówka-Isbell space $\omega \cup A$ whose topology is defined by isolating all the points of $\omega$, and fixing all sets of the form $\{a\} \cup a \backslash F$ where $F$ is finite to be open. By a finite modification of $A$ we mean any family obtained by removing a finite set from each element of $A$. The connection between property (a) and soft almost disjoint families is given by the following theorem.

Theorem 1. Suppose that $A \subseteq[\omega]^{\aleph_{0}}$ is an almost disjoint family. $\Psi(A)$ has property (a) if and only if every finite modification of $A$ is soft.

Proof. Any neighborhood assignment

$$
\left\{U_{a}: a \in A\right\} \text { where } U_{a}=\{a\} \cup\left(a \backslash F_{a}\right)
$$

corresponds to the finite modification $\left\{U_{a} \cap \omega: a \in A\right\}$. For any set $C \subseteq \omega, C$ is closed discrete in $\Psi(A)$, and $C \cap U_{a} \neq \emptyset$ for each $a \in A$ if and only if $C$ has the property that $0<\left|C \cap U_{a}\right|<\omega$ for each $a \in A$.

It is easy to see that there are soft almost disjoint families of every cardinality $\leq 2^{\omega}$ and that no maximal almost disjoint family can be soft. We let $\mathfrak{n} s a$ denote the minimal cardinality of an almost disjoint family that is not soft. Clearly $\mathfrak{n} s a$ is less than or equal to $\mathfrak{a}$, the minimal cardinality of a maximal almost disjoint family. The following question was first raised in [3] though not in the current terminology.

Received by the editors September 15, 2000 and, in revised form, July 25, 2001.

2000 Mathematics Subject Classification. Primary 03E17, 54A25, 54D20.

(a).

Key words and phrases. Almost disjoint families, unbounded sets, weakly separated, property

The author received partial support from NSERC grant 238944. 
Question 1 (Kunen). Is $\mathfrak{n} s a=\mathfrak{a}$ ? If not, is $\mathfrak{n} s a \geq \mathfrak{b}$ ?

If $A, B \subseteq[\omega]^{\aleph_{0}}$, we say that the pair $\langle A, B\rangle$ can be weakly separated if there is an $X \subseteq \omega$ such that $|X \cap b|=\aleph_{0}$ for each $b \in B$ and $|X \cap a|<\aleph_{0}$ for each $a \in A$. Equivalently, by taking the complement of $X$, the pair can be weakly separated iff there is an $X \subseteq \omega$ such that $a \subseteq^{*} X$ for each $a \in A$ and $(\omega \backslash X) \cap b$ is infinite for each $b \in B$. In [1] J. Brendle introduced the following definitions. The almost disjointness principle holds for a cardinal $\kappa$ if for any almost disjoint family $A$ of size $\leq \kappa$ and for any $B \subseteq A$, the pair $\langle B, A \backslash B\rangle$ can be weakly separated. The cardinal $\mathfrak{a} p$ is defined to be the smallest cardinal for which the almost disjointness principle fails. The cardinal ap's relationship to other well known cardinal invariants of the continuum was studied in [1]. In particular, $\mathfrak{p} \leq \mathfrak{a} p \leq \mathfrak{b}$ and it is consistent that both of these inequalities are strict. In this note we prove that $\mathfrak{a} p=\mathfrak{n} s a$, answering both parts of Kunen's question in the negative. The proof that $\mathfrak{n} s a \leq \mathfrak{a} p$ is due to Joerg Brendle and Shunsuke Yatabe. I thank them for allowing me to include their proof here.

$$
\text { 2. } \mathfrak{a} p=\mathfrak{n} s a
$$

The proof splits into two parts. We first present

Theorem 2 (Brendle and Yatabe). $\mathfrak{n} s a \leq \mathfrak{a} p$.

Proof. Let $A$ be an almost disjoint family of size $<\mathfrak{n} s a$. It suffices to prove that the pair $\langle B, A \backslash B\rangle$ can be weakly separated for each $B \subseteq A$. Fix $B \subseteq A$ arbitrary. For each $b \in B$ let $\left\{x_{n}^{b}: n \in \omega\right\}$ be a partition of $b$ into countably many infinite pieces. Consider the family

$$
C=(A \backslash B) \cup\left\{x_{n}^{b}: b \in B, n \in \omega\right\} .
$$

Clearly $C$ is an almost disjoint family of size $<\mathfrak{n} s a$. Thus, there is $X \in[\omega]^{\omega}$ which has finite non-empty intersection with each member of $C$. Then $X$ has finite intersection with each member of $A \backslash B$ and infinite intersection with each member of $B$.

The other inequality is more involved. In [3] the following was proven.

Theorem 3. If $A$ is an almost disjoint family such that $\Psi(A)$ is normal and such that $|A|<\mathfrak{d}$, then $A$ is soft.

Notice that $\Psi(A)$ is normal if and only if for each $B \subseteq A$ the pair $\langle B, A \backslash B\rangle$ can be separated in the sense that there is an $X \subseteq \omega$ such that $b \subseteq^{*} X$ for each $b \in B$ and $a \cap X$ is finite for each $a \in A \backslash B$. The proof of the following theorem is closely related to the proof of Theorem 3

Theorem 4. ap $\leq \mathfrak{n} s a$.

Proof. For a finite sequence $s \in \omega^{n}$ and $k \in \omega, s \frown k \in \omega^{n+1}$ is the sequence extending $s$ whose last entry is $k$. For sequences $s, t \in \omega^{n}$ we write $s \leq t$ if $s(k) \leq t(k)$ for each $k<n$.

Let $A$ be an almost disjoint family of cardinality $<\mathfrak{a} p$. It suffices to prove that $A$ is soft. For each $s \in \omega^{<\omega}$ define $A_{s} \subseteq A$ and sets $X_{s}, Y_{s} \subseteq \omega$ as follows: $A_{\emptyset}=\emptyset$, $X_{\emptyset}=\emptyset$ and $Y_{\emptyset}=\omega$. For each $n \in \omega$ let $A_{\langle n\rangle}=\{a \in A: a \cap n \neq \emptyset\}$. Using the weak separation property fix $X_{\langle n\rangle}$ such that $a \subseteq^{*} X_{\langle n\rangle}$ for each $a \in A_{\langle n\rangle}$. Moreover, $X_{\langle n\rangle}$ is chosen so that $a \cap\left(\omega \backslash X_{\langle n\rangle}\right)$ is infinite for each $a \in A \backslash A_{\langle n\rangle}$. Let $Y_{\langle n\rangle}=\omega \backslash X_{\langle n\rangle}$. 
In addition, we choose these sets so that

(a) $n<m$ implies $X_{\langle n\rangle} \subseteq X_{\langle m\rangle}$ and hence $Y_{\langle m\rangle} \subseteq Y_{\langle n\rangle}$.

Fix $n \geq 1$ and suppose that $A_{s}, X_{s}$ and $Y_{s}$ have been defined for each $s \in \omega^{n}$ so that

(b) $A_{s} \subseteq A$ and $Y_{s}=\omega \backslash X_{s}$ for each $s \in \omega^{\leq n}$.

(c) $a \subseteq^{*} X_{s}$ for each $a \in \bigcup_{i \leq n} A_{s \mid i}$ and $Y_{s} \cap a$ is infinite for each $a \in A \backslash \bigcup_{i \leq n} A_{s \mid i}$.

(d) $X_{s \mid i} \subseteq X_{s \mid j}$ and $Y_{s \mid i} \supseteq \bar{Y}_{s \mid j}$ for each $i<j \leq n$ and for each $s \in \omega^{n}$.

Fix $s \in \omega^{n+1}$. Let

$$
A_{s}=\left\{a \in A \backslash \bigcup_{i \leq n} A_{s \mid i}: a \cap(s(n) \backslash s(n-1)) \cap Y_{s \mid n} \neq \emptyset\right\} .
$$

Using the weak separation property of $A \backslash \bigcup_{i<n} A_{s \mid i}$ restricted to $Y_{s \mid n}$ (this is an almost disjoint family of cardinality $<\mathfrak{a} p)$, fix an $X_{s}^{\prime} \subseteq Y_{s \mid n}$ such that $a \cap Y_{s \mid n} \subseteq^{*} X_{s}^{\prime}$ for each $a \in A_{s}$ and $\left(a \cap Y_{s \mid n}\right) \backslash X_{s}^{\prime}$ is infinite for each $a \in A \backslash \bigcup_{i \leq n+1} A_{s \mid i}$. Let $X_{s}=X_{s \mid n} \cup X_{s}^{\prime}$ and let $Y_{s}=\omega \backslash X_{s}$. It is easy to verify that the recursive hypotheses (b), (c) and (d) are satisfied. Notice that the sets $A_{s}$ satisfy the following properties:

(e) For each $s \in \omega^{n}$ and for each $k>j \geq s(n-1), A_{s \frown j} \subseteq A_{s \frown k}$.

(f) For each $s \in \omega^{n}, A=\bigcup_{i \leq n} A_{s \mid i} \cup \bigcup_{k>s(n-1)} A_{s \frown k}$.

For each $a \in A$ define $f_{a}: \omega \rightarrow \omega$ as follows. Let $f_{a}(0)$ be the minimum $k$ such that $a \in A_{\langle k\rangle}$. For $n>1$, having defined $f_{a} \mid n$ let $f_{a}(n)$ be the minimum $k$ such that for each $s \in \omega^{n}$, if $s \leq f_{a} \mid n$ and $a \notin \bigcup_{i \leq n} A_{s \mid i}$, then $a \in A_{s \frown k}$. Clause (f) and the fact that the set of such $s$ is finite guarantees that $f_{a}(n)$ is well defined.

Using $|A|<\mathfrak{a} p \leq \mathfrak{d}$, fix an increasing $f \in \omega^{\omega}$ such that for each $a \in A$ there is an $n \in \omega$ such that $f(n) \geq f_{a}(n)$.

Claim 5. $A=\bigcup_{n \in \omega} A_{f \mid n}$.

Proof. Fix $a \in A$. Fix $n$ minimal so that $f(n) \geq f_{a}(n)$. Suppose that $a \notin \bigcup_{i \leq n} A_{f \mid i}$. Since $f\left|n \leq f_{a}\right| n, a \in A_{(f \mid n) \frown f_{a}(n)}$ by the definition of $f_{a}(n)$. By clause (e) $a \in A_{(f \mid n) \frown k}$ for each $k \geq f_{a}(n)$ so $a \in A_{f \mid n+1}$.

Now we define $X$ that will witness that $A$ is soft. Let $S_{0}=f(0)$. For each $n>0$ let $S_{n}=(f(n) \backslash f(n-1)) \cap Y_{f \mid n}$ and let $X=\bigcup_{n \in \omega} S_{n}$. Fix $a \in A_{f \mid n+1}$. By definition of $A_{f \mid n+1}, a \cap S_{n} \neq \emptyset$ and by (b) and (d) $a \subseteq^{*} X_{f \mid n+1} \subseteq X_{f \mid m}$ for all $m>n$. By our construction we also have that $X_{f \mid n+1} \cap Y_{f \mid m}=\emptyset$ for each $m>n$. Therefore, $a \cap \bigcup_{m>n} S_{m} \subseteq a \backslash X_{f \mid n+1}$. But since $a \backslash X_{f \mid n+1}$ is finite, $a \cap \bigcup_{m>n} S_{m}$ is finite. Therefore, $0<|a \cap X|<\omega$, as required.

We conclude this section with an example of an almost disjoint family of size $\mathfrak{b}$ that is not soft. In addition to providing an alternate proof of the fact that $\mathfrak{a} p \leq \mathfrak{b}$, the example fails to be soft in a strong way (see the proof of the final claim below). The construction uses a simplification of the set function $H$ introduced by Todorčević to construct S-spaces assuming $\mathfrak{b}=\omega_{1}$ [4].

Theorem 6. There is an almost disjoint family of size $\mathfrak{b}$ that is not soft.

Proof. Fix $B \subseteq \omega^{\omega}$ which is $<^{*}$-unbounded and $<^{*}$-well ordered in type $\mathfrak{b}$. Thus

$$
\left|\left\{f \in B: f<^{*} g\right\}\right|<\mathfrak{b} \text { for each } g \in \omega^{\omega} .
$$

Fix $D=\left\{g_{n}: n \in \omega\right\}$ such that $D \cap B=\emptyset$ and $D$ is dense in $B \cup D$. For each $f \in B$, define

$$
H(f)=\left\{n \in \omega: f\left(\Delta\left(f, g_{n}\right)\right)>n\right\} .
$$


It is clear that if $H(f)$ is infinite, then $\left\{g_{n}: n \in H(f)\right\}$ is a sequence convergent to $f$. So $\{H(f): f \in B$ and $H(f)$ is infinite $\}$ is an almost disjoint family. The following lemma allows us to assume without loss of generality that $H(f)$ is infinite for each $f \in B$.

Lemma 7. The cardinality of $\left\{f \in B:|H(f)|<\aleph_{0}\right\}$ is $<\mathfrak{b}$.

Proof. The following proof is a simple modification of the proof of Lemma 2.0 in [4]. Suppose that $\left\{f \in B:|H(f)|<\aleph_{0}\right\}$ is of cardinality $\mathfrak{b}$. Since $\mathfrak{b}$ is regular, there is a finite $F \subseteq \omega$ such that $B_{0}=\{f \in B: H(f)=F\}$ is of cardinality $\mathfrak{b}$. Therefore, $B_{0}$ is also unbounded. Therefore there are $m \in \omega$ and $s \in \omega^{m}$ such that $\left\{f(m): f \in B_{0}, f \mid m=s\right\}$ is infinite. By density of $D$ we may fix $n \in \omega \backslash F$ such that $g_{n} \mid m=s$. Now fix $f \in B_{0}$ such that $f\left|m=g_{n}\right| m, g_{n}(m) \neq f(m)$ and $f(m)>n$. Thus $n \in H(f)=F$, contradicting $n \notin F$.

The same proof gives the following.

Lemma 8. If $\left\{g_{n}: n \in X\right\}$ is dense in $B \cup\left\{g_{n}: n \in X\right\}$, then $X \cap H(f)$ is infinite for all but $<\mathfrak{b}$ many $f \in B$.

The family $\{H(f): f \in B$ and $H(f)$ is infinite $\}$ may be soft. To get a family that is not soft first fix $\left\{U_{m}: m \in \omega\right\}$, a base for the usual Euclidean topology on $B$. For each $m$, fix $f_{m} \in U_{m} \cap B$ and fix a finite $F_{m} \subseteq H\left(f_{m}\right)$ such that

$$
\left\{g_{n}: n \in H\left(f_{m}\right) \backslash F_{m}\right\} \subseteq U_{m} .
$$

Let $E=\left\{f_{m}: m \in \omega\right\}$. Let

$$
A=\left\{H\left(f_{m}\right) \backslash F_{m}: m \in \omega\right\} \cup\{H(f): f \in B \backslash E\} .
$$

Claim 9. $A$ is not soft.

Proof. If $X \cap\left(H\left(f_{m}\right) \backslash F_{m}\right) \neq \emptyset$ for each $m$, then $\left\{g_{n}: n \in X\right\}$ is dense in $\left\{g_{n}: n \in X\right\} \cup B$. Therefore, by the previous lemma, $X \cap H(f)$ is infinite for all but $<\mathfrak{b}$ many $f \in B$.

\section{Questions}

We will say that the pair $\langle A, B\rangle$ can be separated if there is an $X \subseteq \omega$ such that $|b \backslash X|<\aleph_{0}$ for each $b \in B$ and $|X \cap a|<\aleph_{0}$ for each $a \in A$. So a separated pair is also weakly separated. We will say that an almost disjoint family $A$ is separable if $\langle B, A \backslash B\rangle$ can be separated for each $B \subseteq A$. As discussed above, an almost disjoint family $A$ is separable if and only if the topological space $\Psi(A)$ is normal.

Although it is not a priori obvious, there is a close connection between property (a) and normality in topological spaces (see [2]). Indeed, no example of a normal space of scattered height 2 without property (a) is known. So it is natural to conjecture that every separable almost disjoint family is soft, i.e., that the cardinality restriction in Theorem 3 can be dropped.

We will say that an almost disjoint family is weakly separable if $\langle B, A \backslash B\rangle$ can be weakly separated for each $B \subseteq A$. Clearly any separable almost disjoint family is weakly separable and there are examples of weakly separable not separable almost disjoint families (see [1]). However, the relationship between either of these classes to soft almost disjoint families is open. For example:

Question 2. Is every weakly separable almost disjoint family soft? 
Note that the proof of Theorem 4 yields that if an almost disjoint family $A$ is hereditarily weakly separable (in the sense that $\{a \cap X: a \in A\}$ is weakly separable for any $X \subseteq \omega$ ) and of cardinality $<\mathfrak{d}$, then $A$ is soft. Whether either the hereditary assumption or the cardinality assumption in this result can be dropped is also open.

Note that there is a ZFC example of an almost disjoint family of size $\omega_{1}$ that is not separable. If $\mathfrak{a} p>\omega_{1}$, then this gives a non-separable a.d. family all of whose finite modifications are soft. However, the case for weakly separable is open.

Question 3. Suppose $A$ is an almost disjoint family all of whose finite modifications are soft. Is $A$ weakly separable?

\section{REFERENCES}

[1] J. Brendle Dow's principle and Q-sets, Canadian Mathematical Bulletin, 42 no.1 (1999) 13-24. MR 2000h:03093

[2] W. Just, P.J. Szeptycki and M.V. Matveev Some results on property (a), Topology and its Applications 100 (2000) 67-83. MR 2001j:54019

[3] P.J. Szeptycki and J.E. Vaughan, Almost disjoint families and property (a), Fundamenta Mathematicae 158 (1998) 229-240. MR 99j:54009

[4] S. Todorčević, Partition Problems in Topology, Contemporary Mathematics v 84 American Mathematical Society (1989). MR 90d:04001

School of Analytic Studies and Information Technology, York University, Toronto, Ontario, Canada M3J 1P3

E-mail address: szeptyck@yorku.ca 